\title{
(OPpeneral Surgery \\ A PROSPECTIVE STUDY OF POSTOPERATIVE PULMONARY COMPLICATIONS FOLLOWING UPPER ABDOMINAL SURGERIES
}

\author{
Dr.Harshavardhan Senior resident, Yenepoya Medical College. Deralakatte, Mangalore -575018. \\ Dr. Poovarasan $\mathrm{S}^{*}$ \\ Assistant Professor, Yenepoya Medical College, Deralakatte, Mangalore-575018. \\ *Corresponding Author
}

\section{Dr. Harishchandra}

B

\section{ABSTRACT AI} Professor \& HOD, Yenepoya Medical College, Deralakatte, Mangalore -575018 increased risk of developing post-operative pulmonary complications using a standard preoperative and postoperative evaluation. METHOD:Total of 110 patients admitted with upper abdominal pathologies undergoing elective upper abdominal surgeries in the department of General Surgery,during the period of October 2016 to October 2018 were observed for any pulmonary complications. RESULTS: Study had 51 females and 59 males. 38 patients developed PPC,19 were males and 19 females. The age distribution of these patients are 14 out of 38 patients $(14 \%)$ were in the age group of $21-40$ years, 16 out of 38 patients $(16 \%)$ were in the age group of $41-60$ years, 8 out of 38 patients $(8 \%)$ were in the age group of more than 60 years. Out of 38 patients who developed PPC 15(60.5\%) were smokers and 23(39.5\%) were non-smokers. $21(55.3 \%)$ were underweight,14(36.8\%) were normal weight,2 (5.3\%) were overweight and $1(2.6 \%)$ was class II obesity. $25(65.8 \%)$ had normal preoperative PFT, 6(15.8\%) patients had mild obstructive disease,4(10.5\%) had moderate obstructive disease and $3(7.9 \%)$ had restrictive disease. Among 38 patients with PPC $7(18.4 \%)$ had gastric surgeries, $4(10.5 \%)$ had liver surgeries, $9(23.7 \%)$ underwent surgeries on gall bladder and CBD, 17(44.7\%) had pancreatic surgeries, 1(2.6\%) had surgery on spleen.Among 38 patients who developed PPC all of them had cough with sputum and raised WBC count, 32 had fever, 37 had abnormal breath sounds, 2 had decreased oxygen haemoglobin saturation less than $90 \%, 13(34.2 \%)$ patients had unilateral pleural effusion, $10(26.3 \%)$ had bilateral pleural effusion, $3(7.9 \%)$ had unilateral consolidation and $12(31.6 \%)$ had normal chest $\mathrm{x}$-ray and $11(10 \%)$ had positive sputum culture. CONCLUSION: Patients undergoing upper abdominal surgeries are at a higher risk of developing Postoperative pulmonary complications.Age and Sex are not the significant risk factors for developing PPC.There is a significant correlation between the altered pre-operative pulmonary functions diagnosed by pulmonary function tests and developing PPC.BMI is not a significant risk factor to determine occurrence of PPC.Smoking may not be a significant risk factor to develop PPC.There is a significant relation between the type of upper abdominal surgery and the occurrence of PPC.Cough with sputum ,Fever and Abnormal breath sounds are the most common signs and symptoms seen in patients developing PPC.Pleural effusion is the most common radiological evidence of pulmonary pathology seen in patients with PPC.All patients with postoperative positive sputum culture developed PPC.

KEYWORDS :Postoperative Pulmonary Complications, Upper Abdominal Surgeries

\section{INTRODUCTION}

Pulmonary complications following upper abdominal surgery have a relatively high incidence rate when compared to surgeries performed on other parts of the body ${ }^{1}$. The closer the incision is to the diaphragm, the greater the reduction in postoperative lung volumes ${ }^{1}$.

Post-operative pulmonary complications (PPCs) occur in $5-10 \%$ of patients undergoing non-thoracic surgery and in $22 \%$ of high risk patients. Even in minor surgeries, the incidence can be $1-2 \%$. As many as one in four deaths occurring within a week of surgery is related to pulmonary complications, thus making it the second most common serious morbidity after cardiovascular event ${ }^{3,4}$.

The pulmonary system includes a complex milieu which includes the conducting airways. pulmonary parenchyma. the alveolar capillary interface, an intricate mechanism for control of breathing, complex muscular interplay, and elaborate defense mechanisms. Yet, despite the sophistication of the respiratory system. pulmonary complications are the single most common source of morbidity in the critically ill surgical patient.Postoperative pulmonary complications occur most commonly in those undergoing major abdominal and/or thoracic procedures.Uniformly, these complications result in a predictable set of symptoms including tachypnea, hypoxemia, and hypercapnia followed by respiratory failure each of which are associated with increased morbidity and mortality ${ }^{2}$.

Common postoperative pulmonary complications following general surgery are Pulmonary embolus,obstructive sleep apnoea, ARDS, ventilatorfailure $(\mathrm{PaCO} 2>60 \mathrm{mmHg}, \mathrm{pH}<7.20)$, atelectasis, infe ction-pneumonia and bronchitis,bronchospasm, aspiration of gastric contents, exacerbation of chronic pulmonary disease.Also intraoperative risk factors for postoperative pulmonary complications are thoracic or upper abdominal incisions, general anaesthesia,duration of anaesthesia/surgery,emergent procedures ${ }^{2}$.

\section{AIM AND OBJECTIVE}

The aim of the study is to follow prospectively a group of patients undergoing elective upper abdominal surgeries and to observe those developing pulmonary complications post-opereatively along with factors associated with an increased risk of developing post-operative pulmonary complications using a standard preoperative and postoperative evaluation.

To assess correlation of upper abdominal surgeries along with factors associated with an increased risk of developing post-operative pulmonary complications with regard to postoperative a)Cough with abnormal sputum production b)Temperature above 38degree Celsius c)Abnormal auscultatoryfindings.d)Oxygen hemoglobin saturation less than $90 \%$ e)Raised white cell countf) Radiological evidence of any pulmonary pathology in chestxray(collapse/consolida tion/pleuraleffusion/pneumothorax) (Chest x-ray is done only if patient has respiratory symptoms) g)Positive sputum culture(Sputum culture is done only if patient has respiratory symptoms)

\section{MATERIALS AND METHODS}

- Minimal sample size required is 110 with Prevalence- $20 \%$, Power $=80 \%$, and effect size is 0.12 ,Level of significance $5 \%$ and determined using software $\mathrm{G}^{*}$ power version 3.1

- After ethical committee approval and informed consent, 110 patients undergoing upper abdominal surgeries are followed which includes Gastrectomies, Laparoscopic/Open cholecystectomy, Splenectomy, Whipples procedure and other pancreatic surgeries, Hepatic surgeries, Cystogastrostomy in pseudocyst of pancreas, gastric surgeries with resection and anastomosis such as gastrojejunostomy,also benign and cancer resections on the stomach, liver, pancreas, biliary tree ; CBD exploration, surgeries involving biliary tract, Resection of hydatid cyst in liver, Nissen's fundoplication, Heller's cardiomyotomy for achalasia cardia and Sleeve gastrectomy.

- The source of data was patients in Department of General Surgery at a tertiary care hospital who are admitted and undergoing upper 
abdominal surgeries during the period of October 2016 to October 2018.

- Written informed consent will be taken from Patient Guardian.

- Detailed case will be taken from predesigned proforma. A predesigned Proforma shown below drafted for the study will be used.

- Preoperative and postoperative investigations and tests are done as designed in the proforma.

- A postoperative pulmonary complications was considered when four or more of the following criteria are present: (According to Melbourne Group Scale- MGS) ${ }^{5,6,7}$

- a) Coughwith abnormal sputum production b)Temperature above 38degree Celsiusc)Abnormal auscultatory findings.d)Oxygen hemoglobin saturation less than $90 \%$ e)Raised white cell countf)Radiological evidence of any pulmonary pathology in chest X-ray (collapse/consolidation/pleuraleffusion/pneumothora

- $\mathrm{x})($ Chest $\mathrm{x}$-ray isdone only if patient has respiratory symptoms)g)Positive sputum culture(Sputum culture is done only if patient has respiratory symptoms)

- Patients developing pulmonary complications are identified and recorded.

\section{INCLUSION CRITERIA}

- Age : $>18$ years

- Patients undergoing major(Defined as postoperative hospital stay of $>1$ day) elective(Patients admitted any day before the day of surgery or the night before)upper abdominal surgery in the study period will be included.

- Consecutive patients undergoing upper abdominal surgeries such as Gastrectomies,Laparoscopic/Opencholecystectomy,

- Splenectomy,Whippleprocedure and other pancreatic surgeries, Hepatic surgeries, Cystogastrostomy in pseudocyst of pancreas, gastric surgeries with resection and anastomosis such as gastrojejunostomy,also benign and cancer resections on the stomach, liver, pancreas ,biliary tree, CBD exploration, surgeries involving biliary tract, Resection of hydatid cyst in liver, Nissen'sfundoplication,

- Heller'scardiomyotomy for achalasia cardia and Sleeve gastrectomy were included.

\section{EXCLUSION CRITERIA}

- Emergency upper abdominal surgeries

- History of - chronic cough, wheezing, dyspnoea, haemoptysis, pre-existing pulmonary and /or cardiac disease, COPD, asthma, pulmonary tuberculosis and lung cancer.

\section{RESULTS}

Our study had 110 patients. $3(2.7 \%)$ patients were in the age group of $0-20$ years, $48(43.6 \%)$ were in the age group of $21-40$ years,35(31.8\%) were in age group of 41-60years and $24(21.8 \%)$ were above 60years. Out of these 38 patients developed postoperative pulmonary complications. The age distribution of these patients having pulmonary complications; $0 \%$ in age group of $0-20$ years, 14 out of 38 patients $(14 \%)$ were in the age group of $21-40$ years, 16 out of 38 patients $(16 \%)$ were in the age group of $41-60$ years, 8 out of 38 patients $(8 \%)$ were in the age group of more than 60 years.

Study had 51 females and 59 males. 38 patients developed PPC. Out of 3819 were females $(50 \%)$ and 19 were males $(50 \%)$.

Out of 110 patients $41(62.7 \%)$ were smokers and $69(37.3 \%)$ were nonsmokers.Out of 38 patients who developed PPC 15(60.5\%) were smokers and 23(39.5\%) were non-smokers.

Out of 110 patients 25(22.7\%) were diabetic,14(12.7\%) were hypertensive and $1(0.9 \%)$ patient was a known case of Ischemic heart disease. Out of 38 patients with PPC, 10(26.3\%) were diabetic and $7(18.4 \%)$ were hypertensive.

Among 110 patients 58(52.7\%) patients were underweight, 42(38.25) were normal weight, $7(6.4 \%)$ were overweight, $2(1.8 \%)$ were class I obesity and $1(0.9 \%)$ was class II obesity. Out of 38 patients who developed PPC 21(55.3\%) were underweight, 14(36.8\%) were normal weight, $2(5.3 \%)$ were overweight and $1(2.6 \%)$ was class II obesity.

Out of 110 patients $90(81.8 \%)$ had normal PFT, 9(8.2\%) patients had mild obstructive disease, $4(3.6 \%)$ had moderate obstructive disease and $7(6.4 \%)$ had restrictive disease. All these patients were asymptomatic.Among 38 patients who developed PPC 25(65.8\%) had normal PFT, 6(15.8\%) patients had mild obstructive disease, $4(10.5 \%)$ had moderate obstructive disease and $3(7.9 \%)$ had restrictive disease.fig. 1

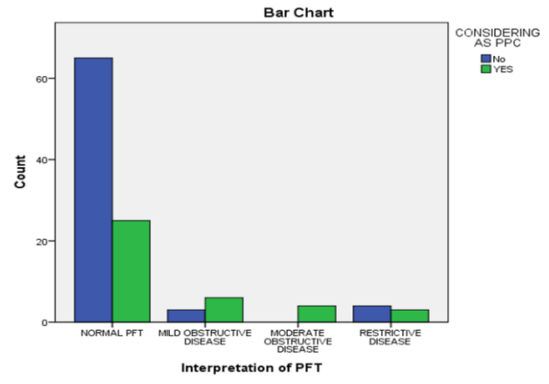

Fig.1 : Relationship between Preoperative PFT and PPC

In 110 patients, $34(30.9 \%)$ patients underwent stomach surgeries(Partial gastrectomy+Gastrojejunostomy,Cystogastrostomy, Triple bypass, Radical gastrectomy, Bariatric surgery, Sleeve gastrectomy,Laparoscopic Nissen's Fundoplication,Heller's Cardiomyotomy), $9(8.2 \%$ ) had surgeries on liver(Excision of hydatid cyst of liver,Hepaticojejunostomy),14(12.7\%) had surgeries on gall bladder and $\mathrm{CBD}$ (Lap converted to open cholecystectomy, CBDexploration,CBDexploration+Cholecystectomy,Cholecystecto my+Choledochojejunostomy+Jejunojejunostomy+HutsonAccessloo $\mathrm{p}+$ Choledocholithotomy, Opencholecystectomy),31(28.2\%) had pancreatic surgeries(Puestow's procedure,Eneucleation of pancreatic tumour,Whipple'sprocedure,Pancreaticojejunostomy+Cystojejunosy omy + Choledochoduodenostomy + cholecystectomy,Distalpancreatect omy + Splenectomy, Laparotomy+Necrosectomy+Peritoneal lavage),3(2.7\%) had sugeries on spleen(Splenectomy) and 19(17.3\%) had laparoscopic surgery on gall bladder(Laparoscopic cholecystectomy)

Among 38 patients with PPC 7(18.4\%) had gastric surgeries,4(10.5\%) had liver surgeries, $9(23.7 \%)$ underwent surgeries on gall bladder and $\mathrm{CBD}, 17(44.7 \%)$ had pancreatic surgeries, $1(2.6 \%)$ had surgery on spleen.fig. 2

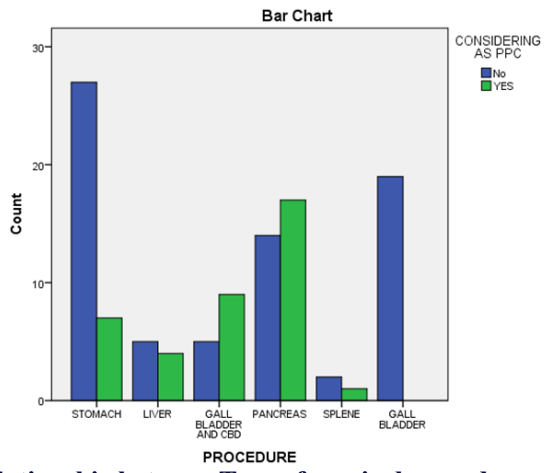

Fig2. : Relationship between Type of surgical procedure and PPC

In 110 patients $52947.3 \%$ ) patients had postoperative cough with sputum.Among 38 patients with PPC all of them had cough with sputum.fig 3 .

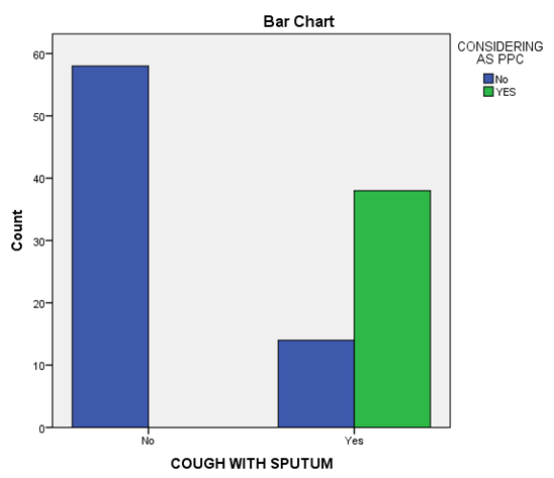

Fig 3. : Postoperative cough with sputum

Among 110 patients $41(37.3 \%)$ had postoperative fever and in 38 patients with PPC 32(84.2\%) developed fever. 
Volume - 10 | Issue - 11 | November - 2020 | PRINT ISSN No. 2249 - 555X | DOI : 10.36106/ijar

Among 110 patients $46(41.8 \%)$ patients had abnormal breath sounds (ronchi,crepitations) postoperatively and in 38 patients who developed PPC 37(97.4\%) had abnormal breath sounds.fig 4.

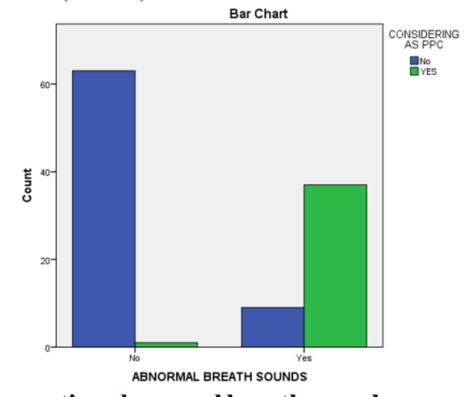

Fig 4. : Postoperative abnormal breath sounds

In 110 patients $2(1.8 \%$ ) had oxygen $\mathrm{Hb}$ saturation less than $90 \%$ and also those 2(5.3\% developed PPC among 38 patients with PPC. Fig 5.

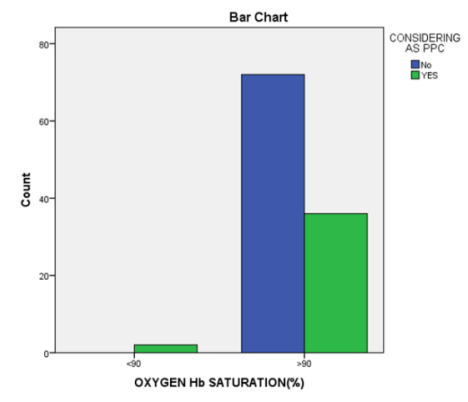

Fig 5. : Postoperative oxygen haemoglobin saturation percentage In 110 patients $60(54.5 \%)$ had increase in total cound and among 38 patients who developed PPC all $38(100 \%)$ had raise in WBC count.

Chest X-ray was done only for the patients who had respiratory symptoms.Among 110 patients chest $\mathrm{x}$-ray was not done for $58(52.7 \%)$ patients. Then $15(13.6 \%)$ patients had unilateral pleural effusion, $10(9.1 \%)$ had bilateral pleural effusion, $3(2.7 \%)$ had unilateral consolidation and $24(21.8 \%)$ had normal chest x-ray. In 38 patients with PPC 13(34.2\%) patients had unilateral pleural effusion, $10(26.3 \%)$ had bilateral pleural effusion,3(7.9\%) had unilateral consolidation and $12(31.6 \%)$ had normal chest $x$-ray.fig 6 .

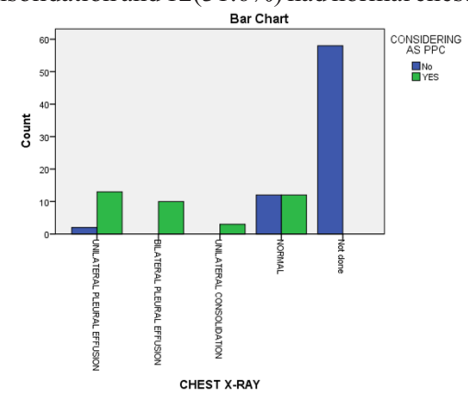

Fig 6. : Postoperative radiological evidence of pulmonary pathology in chest $x$-ray

Sputum culture was done only for the patients who had respiratory symptoms.Among 110 patients $11(10 \%)$ had positive sputum culture and all of them had developed PPC.fig 7.

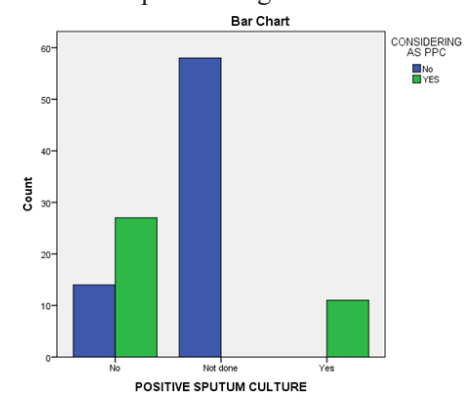

Fig 7 : Postoperative sputum culture

study incidence of postoperative pulmonary complications is $34.5 \%$
38 patients out of 110 developed postoperative pulmonary complications after upper abdominal surgery.

\begin{tabular}{|l|l|l|}
\hline CRITERIA & P VALUE & INFERRENCE \\
\hline Age & .246 & Not significant \\
\hline Gender & .578 & Not significant \\
\hline Smoking & .729 & Not significant \\
\hline Diabetes mellitus & .514 & Not significant \\
\hline Hypertension & .193 & Not significant \\
\hline Ischemic heart disease & .466 & Not significant \\
\hline BMI & .532 & Not significant \\
\hline Pre operative PFT & .003 & Significant \\
\hline Type of surgical procedure & $<.001$ & Significant \\
\hline Post operative Cough with sputum & $<.001$ & Significant \\
\hline Post operative fever & $<.001$ & Significant \\
\hline Post operative abnormal breath sounds & $<.001$ & Significant \\
\hline Post operative spo2 & .049 & Significant \\
\hline Post operative increase in WBC count & $<.001$ & Significant \\
\hline Post operative chest $\mathrm{x}$ ray & $<.001$ & Significant \\
\hline Post operative sputum culture & $<.001$ & Significant \\
\hline
\end{tabular}

\section{DISCUSSION}

A sample of 110 patients were followed to determine the correlation between upper abdominal surgeries and postoperative pulmonary complications.

In our study, among 110 patients 38 patients developed postoperative pulmonary complications.

According to age distribution of the patients in our study pulmonary complications is more in the age groups of $41-60$ years (16 patients) followed by $21-40$ years ( 14 patients) respectively. 8 patients are more than 60years. Age is not of a much significance in our study with a Pvalue of 0.246 .

In the study,both males and females are equally affected with PPC.This shows that both the sex are at equal risk to develop PPC after upper abdominal sugery.Among 38 patients who developed PPC 23 were smokers, with a P- value of 0.729 . This shows smoking may not be a significant risk factor to the occurrence of PPC.But among 41 smokers in the study 15 have developed PPC.There is also no significant relation between chronic diseases such as Diabetes mellitus, Hypertension, Ischemic Heart Disease and the occurrence of PPC as per statistical analysis in our study with a P-value of $0.514,0.193$, 0.466 respectively for each diseases. On comparing BMI with PPC in our study,among the patients who developed PPC 55.3\%(21 patients) of the patients were underwent.But overall BMI is not of a much significance in relation to PPC after upper abdominal surgery in our study with a P-value of 0.532 .Preoperative pulmonary function tests show that there is significant relation between preoperative pulmonary function and PPC with a P-value of 0.003 . Patients with asymptomatic obstructive or restrictive pulmonary diseases are at risk of developing PPC.

In our study PPC occured more among the patients who underwent pancreatic surgeries followed by patients who underwent surgeries of Liver,Gall bladder and CBD which is significant risk factor with a Pvalue of 0.001 . PPC is not observed in the patients who underwent laparoscopic cholecystectomy.Hence significant relation is seen between the type of upper abdomen surgery and the occurrence of PPC.Among the 38 patients with PPC all patients had cough with sputum which is a significant symptom in postoperative patient in our study with a P-value of 0.001 .Fever was also noted in majority of the patients in our study who developed PPC with a significant p-value of 0.001.Almost all patients who developed PPC in our study had abnormal breath sounds like ronchi or crepitations which was a important sign in a patient with PPC with a significant P-value of 0.001.Patients who had decreased oxygen haemoglobin saturation of less than $90 \%$ developed PPC. 2 patients who had decrease in saturation percentage developed PPC which is significant with a Pvalue of 0.049 . This shows that oxygen haemoglobin saturation is one of the indicators to diagnose PPC.There was a elevation in total leucocyte count postoperatively in all the patients with PPC.Total count gives a direct clue to diagnose underlying pulmonary infection in a postoperative patient.Hence total count was a significant indicator in our study with a P-value of 0.001.In our study Chest X-ray was done for the patients who had respiratory symptoms like cough with sputum or abnormal breath breathsounds.Unilateral Pleural effusion was the 
most common pulmonary pathology seen in chest X-ray followed by bilateral pleural effusion being the next common pathology among the patients who developed PPC.Pneumonia was seen in 3 patients postoperatively.But even absence of pleural effusion does not rule out PPC as 12 patients with PPC had no pleural effusion in chest X-ray.But presence of pleural effusion has significant relationship with the PPC with a P-value of 0.001.Sputum culture was sent for the patients who had respiratory symptoms like cough with sputum or abnormal breath breathsounds.Positive sputum culture was seen in $28.9 \%$ of the patients among those who developed PPC.In 110 patients 11 patients had positive sputum culture and all of them developed PPC.

There is a wide variation in the incidence of PPCs from $2 \%$ to $40 \%{ }^{8,9}$. Postoperative pulmonary complications contribute significantly to overall perioperative morbidity and mortality rates.Such complications account for about $25 \%$ of deaths occurring within 6 days of surgery. The frequency rate of these complications varies from 5- $70 \%$. This wide range is due to variations among studies in the definition of postoperative pulmonary complications ${ }^{10}$.

In our study, the incidence of Post-operative pulmonary complications is $34.5 \%$ with variability in patient and procedure related factors.

\section{SUMMARY}

In our two years single institution prospective study,we followed 110 patients who underwent upper abdominal surgeries. The patients included in the study were more than 18 years of age with $54 \%$ males and $46 \%$ females. We found a significant relationship between the upper abdominal surgeries and postoperative pulmonary complications.On the other hand there were patient and procedure related variable factors which are significantly related to the occurrence of postoperative pulmonary complications.

\section{CONCLUSION}

In our study, it was found that:

- Patients undergoing upper abdominal surgeries are at a higher risk of developing Postoperative pulmonary complications.

- Age and Sex are not the significant risk factors for developing PPC.

- There is a significant correlation between the altered pre-operative pulmonary functions diagnosed by pulmonary function tests and developing PPC.

- BMI is not a significant risk factor to determine occurrence of PPC.

- Smoking is not a significant risk factor to develop PPC.

- There is a significant relation between the type of upper abdominal surgery and the occurrence of PPC.

- Cough with sputum ,Fever and Abnormal breath sounds are the most common signs and symptoms seen in patients developing PPC.

- Pleural effusion is the most common radiological evidence of pulmonary pathology seen in patients with PPC.

- All patients with postoperative positive sputum culture developed PPC.

In view of these results, we conclude that postoperative pulmonary complications ntinue to be a significant morbidity following upper abdominal surgeries. Pre-operative risk factors include altered preoperative pulmonary functions, type of surgery and nature of the procedure. Most common postoperative pulmonary complications being cough with sputum, fever, abnormal breath sounds and pleural effusion. Early identification and appropriate treatment goes a long way to tide over the progress to a life threatening state.

\section{REFERENCES}

1. Abraham Mathew,Sharath J.N ,P.T.Thomas, A study of postoperative pulmonary complications following upper abdominal operations, Indian Journal of Basic and Applied Medical Research;December 2015: Vol-5,Issue-1,P.351-358

2. Josef E.Fischer, Daniel B.Jones, et.al, Fischer's Mastery of Surgery by Lippincott Williams and Wilkins,2012,6th edition,chapter-5,Pulmonary risk and Ventilator Support,page 83,86

3. Kelkar, KalpanaVinod. "Post-Operative Pulmonary Complications after NonCardiothoracic Surgery." Indian Journal of Anaesthesia 59.9 (2015): 599-605. PMC.Web. 27 Dec. 2017

4. Fischer SP, Bader AM, Sweitzer BJ. Preoperative evaluation.In Miller's Anaesthesia.7th ed., Ch. 34. Churchill Livingstone. New York. 2010 p. 1019-22.

5. Duration of anaesthesia, type of surgery, respiratory co-morbidity, predicted VO2max Duration of anaesthesia, type of surgery, respiratory co-morbidity, predicted $\mathrm{VO} 2 \mathrm{max}$
and smoking predict postoperative pulmonary complications after upper abdominal surgery: an observational study, Australian Journal of Physiotherapy, Volume 55, Issue 3, 2009, pp. 191-198 Rebecca L. Scholes, Laura Browning, Ewa M. Sztendur, Linda Denehy

6. Agostini P, Cieslik H, Rathinam S, et al Postoperative pulmonary complications following thoracic surgery: are there any modifiable risk factors?,Thorax ;British Medical Journal 2010, 65:815-818.

7. Kumar L, Satheesan KN, Rajan S, Vasu BK, Paul J. Predictors and Outcomes of Postoperative Pulmonary Complications following Abdominal Surgery in a South Indian Population. Anesthesia, Essays and Researches. 2018;12(1):199-205.

8. Kelkar, KalpanaVinod. "Post-Operative Pulmonary Complications after NonCardiothoracic Surgery.” Indian Journal of Anaesthesia 59.9 (2015): 599-605. PMC.Web. 27 Dec. 2017

9. Rock P, Rich PB. Postoperative pulmonary complications.CurrOpinAnaesthesiol 2003;16:123-31

10. AbrahamMathew,Sharath J.N ,P.T.Thomasin Indian Journal of Basic and Applied Medical Research;December 2015: Vol-5,Issue-1,P.351-358 